\title{
ORIGINAL RESEARCH \\ Contrast Extravasation on CT Predicts Mortality in Primary Intracerebral Hemorrhage
}

J. Kim

A. Smith

J.C. Hemphill III

W.S. Smith

Y. Lu

W.P. Dillon

M. Wintermark
BACKGROUND AND PURPOSE: Recent studies of intracerebral hemorrhage (ICH) treatments have highlighted the need to identify reliable predictors of hematoma expansion. The goal of this study was to determine whether contrast extravasation on multisection CT angiography (CTA) and/or contrastenhanced CT (CECT) of the brain is associated with hematoma expansion and increased mortality in patients with primary $\mathrm{ICH}$.

MATERIALS AND METHODS: All patients with primary ICH who underwent CTA and CECT, as well as follow-up noncontrast CT (NCCT) before discharge/death from January 1, 2003, to September 30, 2005, were retrospectively identified. One neuroradiologist reviewed admission and follow-up NCCT for hematoma size and growth. A second neuroradiologist independently reviewed CTA and CECT for active contrast extravasation. Univariate and multivariate logistic regression analyses were performed to evaluate the significance of clinical and radiologic variables in predicting 30-day mortality, designated as the primary outcome. Hematoma growth was considered as a secondary outcome.

RESULTS: Of 56 patients, contrast extravasation was seen in $17.9 \%$ of patients on initial CTA and in $23.2 \%$ of patients on initial CECT following CTA. Univariate analysis showed that the presence of extravasation on $\mathrm{CT}$, large initial hematoma size $(>30 \mathrm{~mL})$, the presence of "swirl sign" on NCCT, the Glasgow Coma Scale and $\mathrm{ICH}$ scores, and international normalized ratio were associated with increased mortality. On multivariate analysis, only contrast extravasation on CT ( $P=.017)$ independently predicted mortality. Contrast extravasation on CT $(P<.001)$ was also an independent predictor of hematoma growth on multivariate analysis.

CONCLUSION: Active contrast extravasation on $\mathrm{CT}$ in patients with primary ICH independently predicts mortality and hematoma growth.
$\mathbf{P}$ rimary intracerebral hemorrhage $(\mathrm{ICH})$ is one of the most devastating forms of stroke, with 30-day mortality rates ranging from $35 \%$ to $44 \% .^{1-3}$ Recent studies of hemostatic treatments such as recombinant activated factor VII as a means to reduce hematoma growth and impact clinical outcome in patients with primary ICH have highlighted the need to identify reliable predictors of hematoma expansion. ${ }^{4-6} \mathrm{~A}$ number of clinical and radiologic variables have been associated with poor outcome following $\mathrm{ICH}$, including age; blood glucose level; Glasgow Coma Scale (GCS) score; and hemorrhage location, size, and intraventricular extension. ${ }^{7-12}$ Several prognostic models for ICH have been developed incorporating both clinical and radiologic variables, among them the ICH score, which includes the GCS score, advanced age, hematoma location, ICH volume, and the presence of intraventricular hemorrhage, to predict 30-day mortality. ${ }^{13}$

Nearly all studies of prognostic variables with respect to imaging have focused exclusively on noncontrast CT (NCCT). One study by Becker et al from $1999^{14}$ examined the role of iodinated contrast administration in primary $\mathrm{ICH}$, concluding that contrast extravasation was independently associated with increased mortality. However, CT technology has evolved considerably since 1999 , primarily due to the intro-

Received August 3, 2007; accepted after revision August 30.

From the Departments of Radiology, Neuroradiology Section (J.K., A.S., Y.L., W.P.D., M.W.), and Neurology (J.C.H.III, W.S.S.), University of California, San Francisco, San Francisco, Calif.

Please address correspondence to Max Wintermark, MD, Department of Radiology, Neuroradiology Section, University of California, San Francisco, 505 Parnassus Ave, Box 0628, San Francisco, CA 94143-0628; e-mail: Max.Wintermark@radiology.ucsf.edu

Indicates article with supplemental on-line tables.

DOI 10.3174/ajnr.A0859 duction of multisection CT scanners. High-quality CT angiography (CTA) and contrast-enhanced CT (CECT) studies of the brain are now routinely performed following contrast administration. The respective roles of CTA and CECT were not separately defined in Becker's study, in which single-section CT scanner technology was used. ${ }^{14}$ A recent study by Goldstein et $\mathrm{al}^{15}$ demonstrated an independent association between contrast extravasation and hematoma expansion but did not explore the relationship with mortality.

The goal of this study was to determine whether contrast extravasation as visualized on multisection CTA and/or CECT is associated with hematoma expansion and increased mortality in patients with primary $\mathrm{ICH}$.

\section{Methods}

\section{Patient Selection}

Our institutional review board approved a retrospective study of patients admitted through the emergency department or directly transferred for ICH between January 2003 and September 2005. All consecutive patients who underwent NCCT, CTA, and CECT of the brain during this timeframe were retrospectively identified. Only patients whose NCCT demonstrated ICH were considered for study inclusion. Patients also should have undergone routine follow-up NCCT before discharge or death to be enrolled in the study. Patients were excluded if ICH was shown by history and diagnostic work-up to be secondary to trauma, aneurysm, vascular malformation, hemorrhagic infarct, vasculitis, venous infarction, Moyamoya disease, or brain tumor.

\section{Clinical Data}

Clinical data were obtained from review of charts and electronic records. Clinical variables examined included age, sex, hypertension, 
diabetes, alcohol use, tobacco use, and hyperlipidemia; systolic blood pressure, diastolic blood pressure, and mean arterial pressure on arrival at our institution; platelet count, activated partial thromboplastin time, and prothrombin time as expressed by the international normalized ratio (INR); time from symptom onset to admission, to CTA, and to follow-up examination; transfer from another hospital or admission from the emergency department; medications (antihypertensives and diuretics including osmotic agents); and surgical procedures such as placement of an external ventricular drain or decompressive craniectomy. GCS and ICH scores were also noted; when they were not specifically recorded in the medical record, they were calculated from the neurologic examination and relevant radiologic data, according to a previously published approach. ${ }^{13}$

In-hospital mortality was recorded. Vital status at 30 days after ICH was also sought in the medical records. If this information was not clearly specified in the medical records, the Internet-based Social Security Death Index was searched (http://ssdi.rootsweb.com); patients alive at hospital discharge, with no mention of death in their medical records and without a recorded date of death in the Internet index, were presumed to be alive 30 days after $\mathrm{ICH}$.

\section{CT}

CT protocol undergone by patients in this study included NCCT, CTA, and CECT and was performed as follows: 1) Unenhanced contiguous axial 3.75-mm-thick NCCT images were obtained through the brain, from the vertex through the skull base, by using $120 \mathrm{kVp}$ and $240 \mathrm{mAs}$. 2) CTA was performed following $70 \mathrm{~mL}$ of iohexol (Omnipaque; GE Healthcare, Piscataway, NJ; $300 \mathrm{mg} \mathrm{I} / \mathrm{mL}$ ) injected intravenously at $4 \mathrm{~mL} / \mathrm{s}$ rate via power injector through an intravenous line, by using the following parameters: $120 \mathrm{kVp} ; 240 \mathrm{mAs}$; section thickness, $1.25 \mathrm{~mm}$; section-acquisition interval, $1 \mathrm{~mm}$; pitch, 1.375:1. Adequate timing of the CTA acquisition was achieved according to a test-bolus technique. 3) CECT was performed 2 minutes after the CTA, by using the same parameters as those used for NCCT.

\section{NCCT Evaluation}

All NCCT scans were reviewed by a neuroradiologist who was blinded to the clinical interpretation of the images and to the clinical condition of the patients. Initial NCCT and the last follow-up NCCT studies available before discharge or death were evaluated at separate sessions; imaging studies were anonymized and randomized so that the reviewer was blinded to patient identity and to whether he was reviewing an admission or follow-up study.

The reviewer was specifically asked the following: 1) to assess the location of hemorrhage (supratentorial or infratentorial), and 2) to measure the volume of hemorrhage in milliliters by using the $A B C / 2$ method, ${ }^{16}$ where $A$ is the greatest diameter of hemorrhage on the CT section with the largest area of hemorrhage, $B$ is the diameter perpendicular $\left(90^{\circ}\right)$ to $A$, and $C$ is the number of sections with hemorrhage multiplied by the section thickness. ${ }^{16} \mathrm{C}$ was calculated by a comparison of each CT section with hemorrhage with the CT section demonstrating the largest area of hemorrhage on that scan. If the area of hemorrhage for a particular section measured at least $75 \%$ of the largest area of hemorrhage, that section was considered 1 hemorrhage section for determining $C$; if the area was approximately $25 \%-75 \%$ of the largest area, the section was considered half a hemorrhage section; and if the area was $<25 \%$ of the largest area of hemorrhage, the section was not considered a hemorrhage section. These CT hemorrhage section values were then added and multiplied by the individual section thickness $(0.375 \mathrm{~cm})$ to determine the value for $C .{ }^{16}$ The volume of hemorrhage was considered both as a continuous and dichotomous variable, with $30 \mathrm{~mL}$ selected as a threshold to distinguish between "small" and "large" hematomas, as done in prior studies. ${ }^{17,18}$

The reviewer was also asked: 3 ) to determine whether a "swirl sign" was present on initial NCCT because hypoattenuated areas within a high-attenuation hematoma or "swirl sign" could potentially indicate ongoing hemorrhage. ${ }^{19}$ The reviewer was also asked, 4) to assess the presence or absence of intraventricular hemorrhage; 5) to record the presence or absence of hydrocephalus, with severity of the hydrocephalus being graded as mild (dilation of the third ventricle and temporal horns of lateral ventricles), moderate (dilation of all ventricles), and severe (transependymal flow); 6) to assess the presence or absence of herniation (subfalcine, transtentorial, tonsillar, and/or transcalvarial); 7) and to record the presence or absence of craniectomy and/or external ventricular drain.

\section{CTA and CECT Evaluation}

CTA and CECT were reviewed by a second neuroradiologist who was also blinded to the clinical interpretation of the images and to the clinical condition of the patients. This reviewer performed his review independently of the first reviewer and evaluated CTA and CECT images separately from each other (images were anonymized and randomized so that it was impossible to associate the CTA and CECT scans). This reviewer was specifically asked the following: 1 ) to assess the presence or absence of active contrast extravasation, as evidenced by high-attenuation contrast material within the hematoma, and 2) to note the number of foci of active contrast extravasation, if present.

After evaluation of all CTA and CECT images, a composite CTA/ CECT score was calculated, with zero equal to no areas of extravasation, 1 equal to extravasation on either CTA or CECT, and 2 equal to extravasation on both CTA and CECT.

\section{Outcome Measurements and Statistical Analysis}

Thirty-day mortality was the primary outcome measure. Hospital mortality was evaluated as a separate outcome. Hematoma growth on NCCT between admission and immediately before discharge or death (considered as a continuous variable) was used as a secondary outcome.

Univariate analyses for association of clinical and imaging variables with respect to primary and secondary outcomes were performed with $\chi^{2}$ test statistics for dichotomous variables and Wilcoxon rank sum tests for continuous data. Predictive variables with $P$ values $<.10$ in univariate analysis were retained and included in a multivariate backward stepwise logistic regression model; for this multivariate model, the significance threshold was set at .05 .

\section{Results}

\section{Patient, Clinical, and Radiologic Data}

Of all patients who had undergone NCCT/CTA/CECT from January 1, 2003, to September 30, 2005, 56 patients had primary $\mathrm{ICH}$ and follow-up imaging and were included in our study (Fig 1). Median time from symptom onset to admission NCCT/CTA/CECT was 13 hours (interquartile range, 7.2525.5 hours). Median time from admission NCCT/CTA/CECT to follow-up CT scanning was 2.4 days (interquartile range, $1.0-9.4$ days).

Clinical and radiologic data collected in patients from our series are summarized in the On-line Table. A longer INR was 


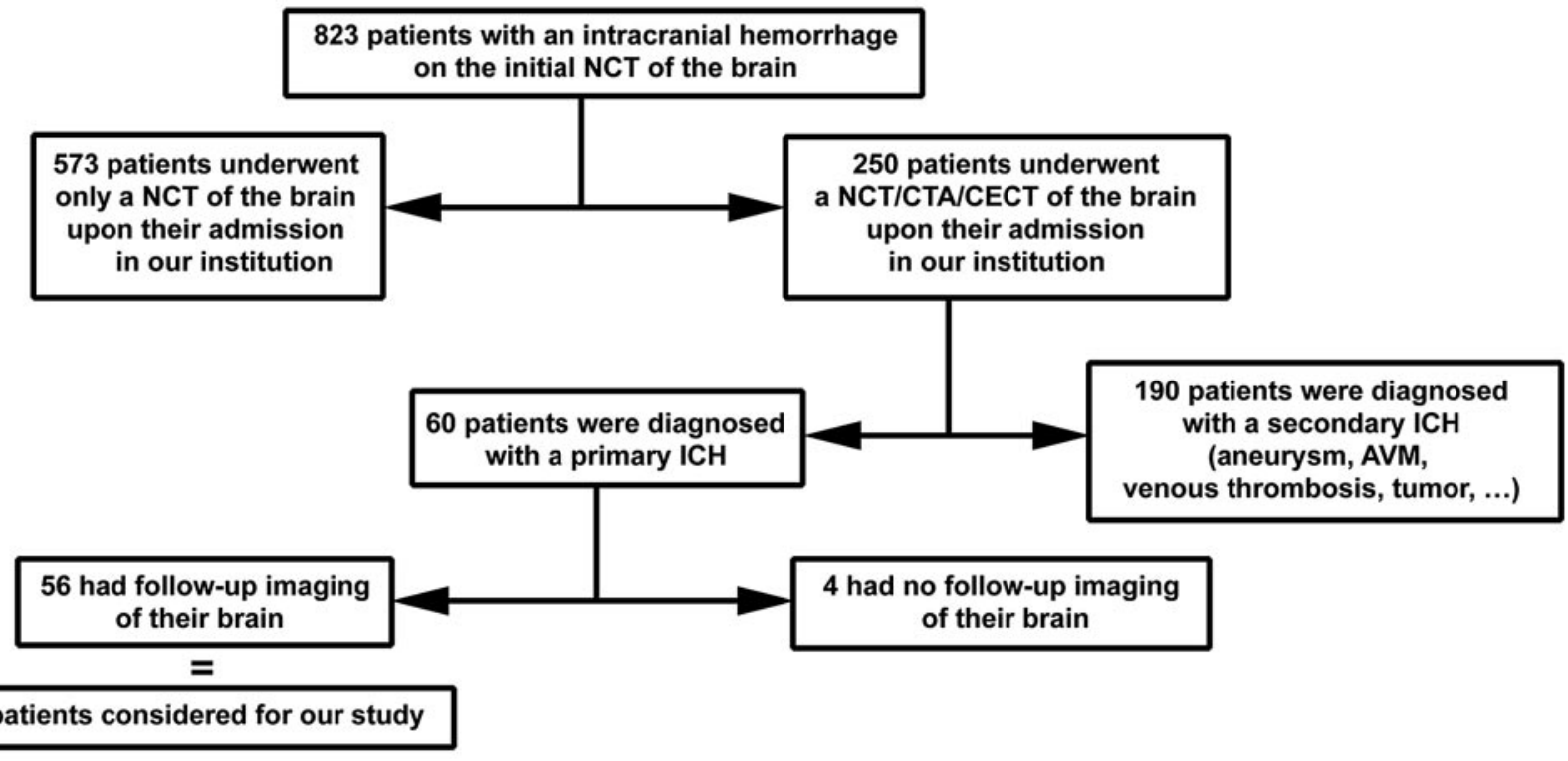

Fig 1. Schematic of patient selection for our study. The decision of obtaining a CTA/CECT of the brain immediately following the initial NCCT was made by the neurologist in charge of the patient, on the basis of the degree of suggestion of an underlying origin for the intracranial hemorrhage (ICH). Seventy-six percent (190/250) turned out to have such an underlying cause (aneurysm, vascular malformation, vasculitis, venous infarction, Moyamoya disease, or brain tumor), whereas $24 \%$ (60/250) were finally diagnosed with primary ICH. Among the 60 patients with primary ICH, 56 underwent follow-up brain imaging. Among the 4 who did not, 2 died before follow-up imaging. AVM indicates arteriovenous malformation; NCT, noncontrast CT.
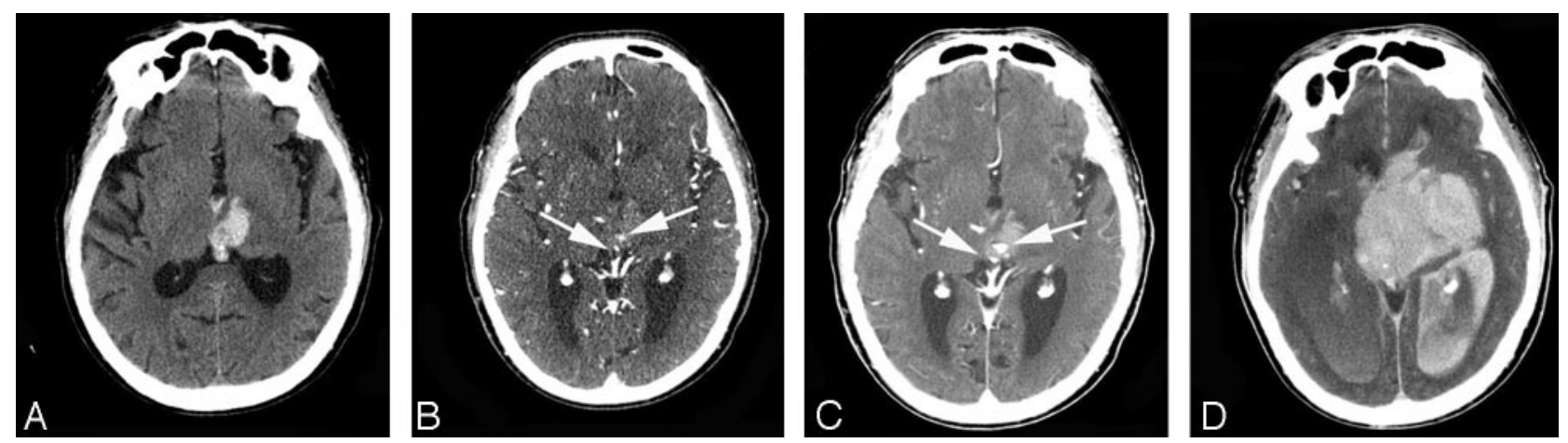

Fig 2. A 69-year-old man underwent imaging 2 hours following onset of right-sided paralysis. A, Admission NCCT demonstrates left thalamic hematoma with extension of hemorrhage into the third ventricle. Admission CTA $(B)$ and CECT (C), respectively, show 2 foci of active extravasation (arrows). D, Follow-up NCCT 12 hours later shows marked hematoma growth with hemorrhage in both lateral ventricles and severe hydrocephalus. The patient had a fatal outcome.

associated with increased mortality in univariate analysis, but this association did not hold in the multivariate analysis.

\section{CTA and CECT Extravasation}

Active extravasation was seen in $17.9 \%$ of patients (10/56) on CTA and in $23.2 \%$ of patients (13/56) on CECT. The number of foci of active extravasation ranged from 1 to 2 on CTA ( 5 patients had 1 focus of extravasation and 5 patients had 2 foci) and from 1 to 7 on CECT ( 5 patients had 1 focus of extravasation, 3 patients had 2 foci, 1 patient had 3,1 patient had 4,1 patient had 5, 1 patient had 6 , and 1 patient had 7). Figure 2 depicts the imaging findings of a patient with $\mathrm{ICH}$ with extensive contrast extravasation on both CTA and CECT, with the follow-up CT showing a significant increase in the size of the parenchymal hematoma. CECT demonstrated 5 cases of extravasation not seen on CTA (Fig 3), whereas CTA showed 2 cases of extravasation not appreciated on CECT (Fig 4); the remaining 8 cases of extravasation were seen on both CTA/CECT.
There was a significantly shorter time from symptom onset to CTA/CECT in patients with active extravasation (10.6 \pm 13.1 hours) than in patients without active extravasation (37.6 \pm 52.8 hours) (2-tailed $t$ test, $P=.004)$. However, active extravasation was seen up to 24 hours after symptom onset on CTA; on CECT, active extravasation was seen up to 48 hours after symptom onset.

Initial hematoma volume was $28.5 \mathrm{~mL}$ (range, 0.4-109.5 $\mathrm{mL}$ ) in patients with active extravasation and $21.6 \mathrm{~mL}$ (range, $0.3-136 \mathrm{~mL}$ ) in patients without active extravasation (no significant difference, $P=.518$ ). Final hematoma volume was $42.0 \mathrm{~mL}$ (range, $6.0-111.0 \mathrm{~mL}$ ) in patients with active extravasation, which was significantly higher $(P=.033)$ than that in patients without active extravasation (mean, $23.0 \mathrm{~mL}$; range, 0.9-138.6 mL). Time from admission NCCT/CTA/CECT to follow-up CT scanning was not significantly different $(P=$ .610 ) in patients with active extravasation (median, 2.0 days; interquartile range, 1.2-6.3 days) and without active extravasation (median, 2.6 days; interquartile range, 0.9-10.5 days). 

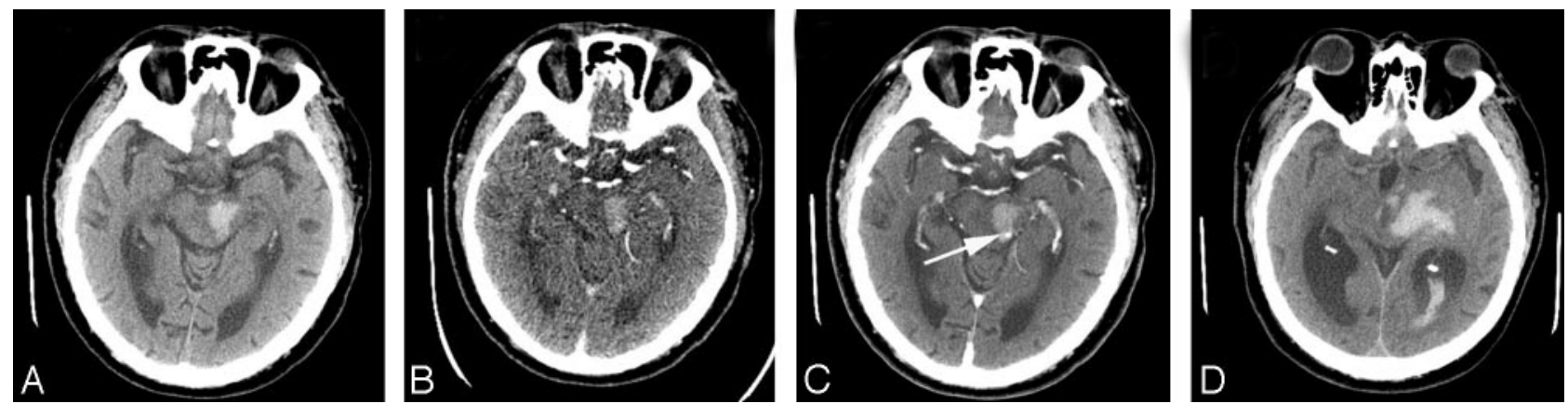

Fig 3. An 82-year-old man underwent imaging 0.5 hours following onset of right-sided weakness. Admission NCCT $(A)$ and CTA $(B)$ demonstrate hemorrhage in the left midbrain without active extravasation on CTA. C, Admission CECT, however, reveals focal high attenuation (arrow) in the left tectal plate, consistent with active extravasation. D, Follow-up NCCT 17 hours later shows hematoma expansion into the left thalamus and lateral ventricle with marked hydrocephalus. The patient had a fatal outcome.


Fig 4. An 85-year-old woman underwent imaging 1 hour following onset of left-sided weakness. $A$, Admission NCCT demonstrates hemorrhage in the right superior frontal gyrus. $B$, Admission CTA conspicuously reveals a focal area of high attenuation (arrow) within the hematoma consistent with active extravasation. $C$, Admission CECT shows a slightly heterogeneous right frontal hematoma with several areas of higher attenuation, frequently seen with acute hemorrhage, which were not interpreted by the reviewer as discrete foci of contrast extravasation (as a reminder, the reviewer evaluated CTA and CECT images separately from each other). This patient survived.

\section{Comparison of Mortality Rates in Patients with and without Extravasation}

The overall 30 -day mortality rate was $28.6 \%(16 / 56)$. The 30-day mortality rate of patients with active extravasation on CTA or CECT was $53.3 \%$ (8/15); this was significantly higher $\left(\chi^{2}, P=.013\right)$ than the 30 -day mortality rate of patients without active extravasation on CTA and CECT, which was $19.5 \%(8 / 41)$. The number of foci of extravasation on CTA or CECT did not influence mortality or hematoma growth on multivariate analysis. Similarly, the composite CTA/CECT score described in the "Methods" section did not add significant information compared with the simple presence or absence of contrast extravasation on either CTA or CECT.

\section{Comparison of Clinical and Radiologic Variables, Including CTA/CECT Contrast Extravasation, with Respect to Mortality (Primary Outcome)}

The results of the univariate analysis for the association between all evaluated clinical and radiologic variables and 30-day mortality are summarized in the On-line Table. Active extravasation on CTA and/or CECT, "swirl sign" on NCCT, large initial hematoma size $(>30 \mathrm{~mL})$, GCS score, ICH score, and INR were significantly associated with 30-day mortality $(P<.10)$ and were considered for the multivariate analysis.
Because the ICH score was a summed ordinal variable and included hematoma size and the GCS score, we decided to consider the components of the ICH score, rather than the ICH score itself, in the multivariate analysis to avoid collinearity. The variables included in the multivariate backward stepwise logistic regression model were active extravasation on CTA and/or CECT, "swirl sign" on NCCT, large initial hematoma size, infratentorial location of the hematoma, presence of intraventricular hemorrhage, GCS score, INR, age, and time from symptom onset to initial NCCT/CTA/CECT at our institution. The backward stepwise process successively dropped infratentorial location $(P=.716)$, GCS score $(P=.373)$, age $(P=.327)$, NCCT "swirl sign" $(P=.432)$, time from symptom onset to initial NCCT/CTA/CECT at our institution $(P=.366)$, INR $(P=$ $.163)$, large hematoma size $(P=.126)$, and intraventricular hemorrhage $(P=.062)$. Only the presence of active extravasation on CTA and/or CECT remained significant in prediction of death (odds ratio, 4.71; 95\% confidence interval, 1.31-16.87; $P=.017$ ).

The same results were obtained when hospital mortality instead of 30-day mortality was used as the outcome because only 1 patient alive at hospital discharge was found to be deceased within 30 days of ICH. 
Comparison of Clinical and Radiologic Variables, Including CTA/CECT Contrast Extravasation, with Respect to Hematoma Growth (Secondary Outcome)

Univariate analysis of all evaluated clinical and radiologic variables with respect to hematoma growth between initial and follow-up imaging demonstrated that active extravasation on CTA $(P=.004)$ and CECT $(P=.048)$, number of foci of extravasation on CTA $(P=.004)$ and CECT $(P=.045)$, composite CTA/CECT score $(P=.048)$, ICH score $(P=.004)$, initial hematoma volume $(P=.005)$, and herniation $(P=$ $.044)$ predicted hematoma growth. However, only extravasation on CECT remained significant on multivariate analysis $(P<.001)$.

\section{Patients Admitted Through the Emergency Department and Patients Transferred from Outside Hospitals}

Because our institution is a tertiary care referral center, 32 of 56 patients in the study were transferred from outside hospitals. Transferred patients underwent admission NCCT/CTA/ CECT after a median time of 23 hours (interquartile range, 12.5-48 hours), which was significantly longer (Wilcoxon rank sum test, $P<.001$ ) compared with patients who were admitted from the emergency department with a median time of 5.3 hours from symptom onset to admission NCCT/CTA/ CECT (interquartile range, 2-10 hours). Transferred patients also had significantly larger volumes of hemorrhage on initial NCCT $(31.7 \pm 33.1 \mathrm{~mL})$ compared with patients admitted from the emergency department $(14.7 \pm 13.7 \mathrm{~mL})$ (Wilcoxon rank sum test, $P<.001)$. On the other hand, patients admitted from the emergency department $(10 / 24$ or $41.7 \%)$ had a significantly higher incidence of active extravasation on CTA or CECT as compared with transferred patients $(5 / 32$ or $15.6 \%$; $\left.\chi^{2}, P=.029\right)$. However, there were no significant differences between the 2 groups in terms of mortality $\left(\chi^{2}, P=.794\right)$, the need for external ventricular drain $\left(\chi^{2}, P=.520\right)$, or craniectomy $\left(\chi^{2}, P=.380\right)$.

\section{Interpretation}

Recent studies of hemostatic treatments such as recombinant activated factor VII as a means to reduce hematoma growth and improve clinical outcome in patients with primary $\mathrm{ICH}$ have highlighted the need to identify reliable predictors of hematoma expansion. ${ }^{4-6}$ Nearly all previous outcome studies of spontaneous ICH have focused on the evaluation of NCCT studies of the brain. Prognostic models for outcome in patients with ICH most frequently include the initial hematoma size as assessed on NCCT in addition to clinical variables such as the GCS score, which have been found to be significant independent predictors of mortality in multiple studies. ${ }^{13,20-22}$

The present study demonstrated that the addition of intravenous contrast in the setting of acute $\mathrm{ICH}$, usually performed to assess an underlying cause or substrate of hemorrhage, can provide useful information about the risk of hematoma expansion in acute ICH. This is in agreement with the recent study by Goldstein et al. ${ }^{15}$ Also, contrast extravasation on CTA/CECT independently predicted hospital mortality and 30-day mortality. Although ICH score and INR correlated significantly with mortality as on previous studies, our study found that contrast extravasation on CT was the only independent predictor of mortality on multivariate analysis.
Focal contrast accumulation, which we assume to represent active extravasation of contrast, was seen in $17.9 \%$ of patients on CTA and in $23.2 \%$ of patients on CECT. We found that patients with active extravasation on CTA/CECT had significantly shorter mean times to imaging than patients without extravasation. However, time from symptom onset to initial NCCT/CTA/CECT at our institution, if significant in the univariate analysis, did not prove to be an independent predictor of mortality in the multivariate analysis. Also, most interesting, contrast extravasation on CT was seen up to 48 hours following symptom onset, suggesting that hematoma growth may continue to occur later into the course of $\mathrm{ICH}$, though hematoma growth is traditionally considered to occur mainly during the first few hours following onset. ${ }^{17,18,23}$ The observation of active extravasation up to 48 hours after symptom onset in this study may carry implications for designing the treatment window to be used in further ICH treatment trials.

Contrast extravasation on CT was more frequently seen on CECT compared with CTA, possibly due to more time for contrast pooling on CECT. However, there were cases in which extravasation was seen on CTA only, perhaps as a result of a higher rate of contrast extravasation. Hence, reviewing both CTA and CECT images is important because each showed cases of extravasation not seen on the other. Both carried the same information in terms of predicting hematoma growth and mortality.

Limitations of this study include the relatively small sample size. Also, a significant number of patients were transferred from another hospital, and contrast extravasation on CTA/ CECT may have been underestimated in these transferred patients with longer times to imaging. Comparison with follow-up imaging for hematoma growth was made on the last CT scan available before discharge or death. We did not standardize the follow-up interval so that the impact of contrast extravasation on ultimate hematoma size could be evaluated, but lack of a uniform follow-up interval may have misrepresented hematoma growth, potentially in cases of patients who were hospitalized for extensive periods of time with gradual hematoma resorption. Finally, acquisition of CTA/CECT in patients with $\mathrm{ICH}$ was not performed according to specific guidelines or protocol; patients in whom an underlying cause for hemorrhage was suspected generally underwent CTA/ CECT, whereas patients with classic clinical and radiologic findings for primary hypertensive hemorrhage may not have been administered contrast on admission. This has likely influenced the types of patients included in this study and its generalizability. However, because the ordering of a CTA/ CECT was not related to the presence or absence of active contrast extravasation, there is no reason to suspect a bias that would have compromised the internal validity of our study. The "swirl sign" on NCCT was associated with increased mortality in univariate analysis, but this association did not hold in the multivariate analysis.

\section{Conclusion}

Intravenous contrast material should be administered to patients with ICH not only to evaluate underlying sources of hemorrhage but also to identify patients at risk for hematoma expansion and death. Assessing a simple radiologic feature 
such as contrast extravasation on CT may serve as a very efficient and straightforward method of risk-stratifying patients on admission. This should be confirmed in prospective studies in which all patients with ICH would systematically undergo CTA and CECT.

\section{References}

1. Fogelholm R, Avikainen S, Murros K. Prognostic value and determinants of first-day mean arterial pressure in spontaneous supratentorial intracerebral hemorrhage. Stroke 1997;28:1396-400

2. Godoy DA, Pinero G, Di Napoli M. Predicting mortality in spontaneous intracerebral hemorrhage: can modification to original score improve the prediction? Stroke 2006;37:1038-44

3. Broderick JP, Brott TG, Duldner JE, et al. Volume of intracerebral hemorrhage: a powerful and easy-to-use predictor of 30-day mortality. Stroke 1993; 24:987-93

4. Juvela S, Kase CS. Advances in intracerebral hemorrhage management. Stroke 2006;37:301-04

5. Mayer SA, Brun NC, Begtrup K, et al. Recombinant activated factor VII for acute intracerebral hemorrhage. $N$ Engl J Med 2005;352:777-85

6. Brown DL, Morgenstern LB. Stopping the bleeding in intracerebral hemorrhage. N Engl J Med 2005;352:828-30

7. Davis SM, Broderick J, Hennerici M, et al. Hematoma growth is a determinant of mortality and poor outcome after intracerebral hemorrhage. Neurology 2006;66:1175-81

8. Juvela S. Risk factors for impaired outcome after spontaneous intracerebral hemorrhage. Arch Neurol 1995;52:1193-200

9. Franke CL, van Swieten JC, Algra A, et al. Prognostic factors in patients with intracerebral haematoma. J Neurol Neurosurg Psychiatry 1992;55:653-57

10. Gebel JM Jr, Jauch EC, Brott TG, et al. Relative edema volume is a predictor of outcome in patients with hyperacute spontaneous intracerebral hemorrhage. Stroke 2002;33:2636-41

11. Jauch EC, Lindsell CJ, Adeoye O, et al. Lack of evidence for an association between hemodynamic variables and hematoma growth in spontaneous intracerebral hemorrhage. Stroke 2006;37:2061-65

12. Daverat P, Castel JP, Dartigues JF, et al. Death and functional outcome after spontaneous intracerebral hemorrhage: a prospective study of 166 cases using multivariate analysis. Stroke 1991;22:1-6

13. Hemphill JC 3rd, Bonovich DC, Besmertis L, et al. The ICH score: a simple, reliable grading scale for intracerebral hemorrhage. Stroke 2001;32:891-97

14. Becker KJ, Baxter AB, Bybee HM, et al. Extravasation of radiographic contrast is an independent predictor of death in primary intracerebral hemorrhage. Stroke 1999;30:2025-32

15. Goldstein JN, Fazen LE, Snider R, et al. Contrast extravasation on CT angiography predicts hematoma expansion in intracerebral hemorrhage. Neurology 2007;68:889-94

16. Kothari RU, Brott T, Broderick JP, et al. The ABCs of measuring intracerebral hemorrhage volumes. Stroke 1996;27:1304-05

17. Kazui S, Minematsu K, Yamamoto $\mathrm{H}$, et al. Predisposing factors to enlargement of spontaneous intracerebral hematoma. Stroke 1997;28:2370-75

18. Fujii Y, Takeuchi S, Sasaki O, et al. Multivariate analysis of predictors of hematoma enlargement in spontaneous intracerebral hemorrhage. Stroke 1998;29:1160-66

19. Al-Nakshabandi NA. The swirl sign. Radiology 2001;218:433

20. Tuhrim S, Horowitz DR, Sacher M, et al. Validation and comparison of models predicting survival following intracerebral hemorrhage. Crit Care Med 1995;23:950-54

21. Tuhrim S, Dambrosia JM, Price TR, et al. Intracerebral hemorrhage: external validation and extension of a model for prediction of 30-day survival. Ann Neurol 1991;29:658-63

22. Portenoy RK, Lipton RB, Berger AR, et al. Intracerebral haemorrhage: a model for the prediction of outcome. J Neurol Neurosurg Psychiatry 1987;50:976-79

23. Brott T, Broderick J, Kothari R, et al. Early hemorrhage growth in patients with intracerebral hemorrhage. Stroke 1997;28:1-5 